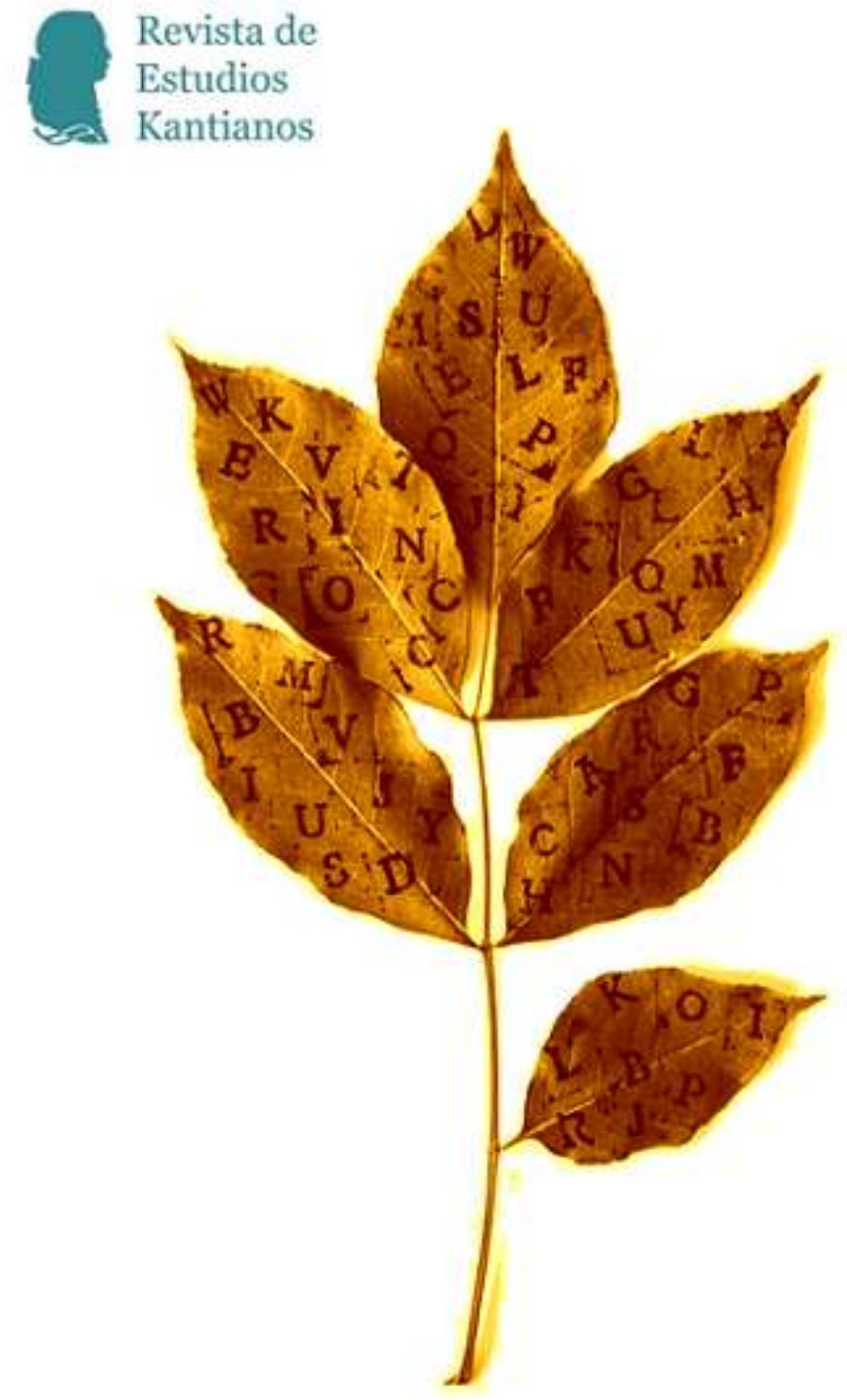




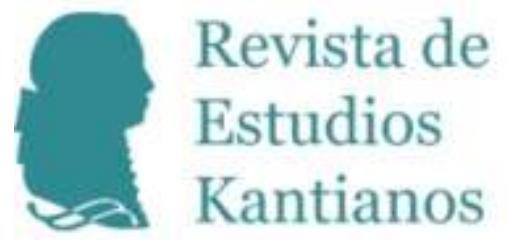




\section{Revista de Estudios Kantianos}

Publicación internacional de la Sociedad de Estudios Kantianos en Lengua Española Internationale Zeitschrift der Gesellschaft für Kant-Studien in Spanischer Sprache International Journal of the Society of Kantian Studies in the Spanish Language

\section{Dirección}

Pedro Jesús Teruel, Universitat de València

pedro.teruel@uv.es

Hernán Pringe, CONICET-Universidad de Buenos Aires/

Universidad Diego Portales, Santiago de Chile

hpringe@gmail.com

\section{Secretario de edición}

Fernando Moledo, Universidad de Buenos Aires - CONICET

fernandomoledo@gmail.com

\section{Secretaria de calidad}

Marcela García, Instituto de Investigaciones Filosóficas, UNAM

garciar.marcela@gmail.com

\section{Editores científicos}

Jacinto Rivera de Rosales, UNED, Madrid

Claudia Jáuregui, Universidad de Buenos Aires

Vicente Durán, Pontificia Universidad Javeriana, Bogotá

Julio del Valle, Pontificia Universidad Católica del Perú, Lima

Jesús Conill, Universitat de València

Gustavo Leyva, Universidad Autónoma de México, México D. F.

María Xesús Vázquez Lobeiras, Universidade de Santiago de Compostela

Wilson Herrera, Universidad del Rosario, Bogotá

Pablo Oyarzun, Universidad de Chile, Santiago de Chile

Paula Órdenes Azúa, Universität Heidelberg 


\section{Comité científico}

Juan Arana, Universidad de Sevilla

Reinhardt Brandt, Philipps-Universität Marburg

Mario Caimi, Universidad de Buenos Aires

Monique Castillo, Université de Paris-Est

Adela Cortina, Universitat de València

Bernd Dörflinger, Universität Trier

Norbert Fischer, Universität Eichstätt-Ingolstadt

Miguel Giusti, Pontificia Universidad Católica del Perú

Dulce María Granja, Universidad Nacional Autónoma de México

Christian Hamm, Universidad Federal de Santa María, Brasil

Dietmar Heidemann, Université du Luxembourg

Otfried Höffe, Universität Tübingen

Claudio La Rocca, Università degli Studi di Genova

Juan Manuel Navarro Cordón, Universidad Complutense, Madrid

Carlos Pereda, Universidad Nacional Autónoma de México

Gustavo Pereira, Universidad de la República, Uruguay

Ubirajara Rancan de Azevedo, Universidade Estadual Paulista, Brasil

Margit Ruffing, Johannes Gutenberg-Universität Mainz

Gustavo Sarmiento, Universidad Simón Bolívar, Venezuela

Sergio Sevilla, Universitat de València

Roberto Torretti, Universidad Diego Portales, Santiago de Chile

Violetta Waibel, Universität Wien

Howard Williams, University of Aberystwyth

Allen W. Wood, Indiana University

Diseño, revisión de estilo y maqueta

Josefa Ros Velasco, Universidad Complutense de Madrid

\section{Entidades colaboradoras}

Sociedad de Estudios Kantianos en Lengua Española (SEKLE)

Departament de Filosofia de la Universitat de València 


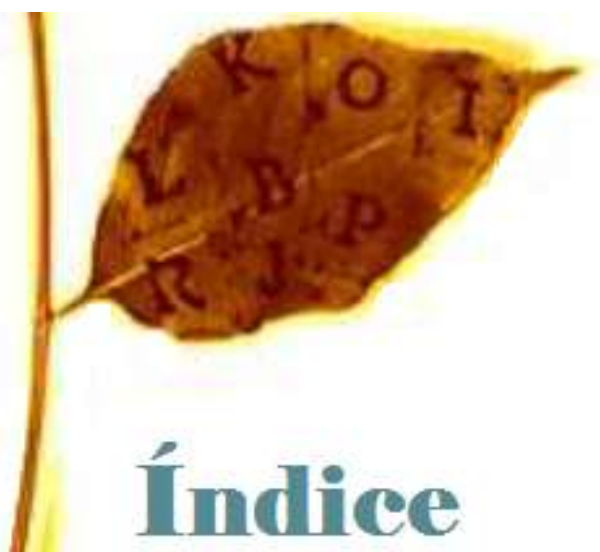

\section{Artículos}

113 ¿"El yo propiamente tal” (I. Kant) o "el originario ser sí mismo” (D. Heinrich)? Sobre algunas características del concepto kantiano de autoconciencia

Heiner Klemme

DOI 10.7203/REK.2.2.9068

126 La noción kantiana de verdad transcendental

Stéfano Straulino Torre

DOI 10.7203/REK.2.2.8809

146 Una travesía kantiana a través del Escila constructivista y el Caribdis realista. Apuntes para un abordaje kantiano-constitutivista de las fuentes de la normatividad

Martín Fleitas González

DOI 10.7203/REK.2.2.8810

174 El malestar kantiano. Filosofía y ciencia al encuentro con la naturaleza Juan Felipe Guevara

DOI 10.7203/REK.2.2.8812

191 ¿Puede haber una fundamentación kantiana de los derechos humanos? Algunas consideraciones críticas

Nuria Sánchez Madrid

DOI 10.7203/REK.2.2.8871

\section{Semblanza}

208 El pensamiento filosófico de Ernesto Mayz Vallenilla

Gustavo Sarmiento

DOI 10.7203/REK.2.2.9021 


\section{Reseñas}

217 Claudia Jáuregi, Fernando Moledo, Hernán Pringe y Marcos Thisted (eds.) Crítica y Metafísica. Homenaje a Mario Caimi. Studien und Materialen zur Geschichten der Philosophie. Hildesheim, Olms, 2015, 460 pp. ISBN: 978-3-487-15237-0.

Alba Jiménez Rodríguez

DOI 10.7203/REK.2.2. 8814

227 Fernando Moledo: Los años silenciosos de Kant: Aspectos de la génesis de la Deducción Trascendental en la década de 1770. Seguido de la traducción del Legado de Duisburg (ca. 1775). Buenos Aires, Prometeo, 2014, 192 pp. ISBN: 978-987-574-667-1.

Pablo Moscón DOI 10.7203/REK.2.2. 8830

231 Salvi Turró: Filosofia i Modernitat. La reconstrucció de l'ordre del món. Barcelona, Edicions Universitat de Barcelona, 2016, 228 pp. ISBN: 978-84-475-3966-6.

Pedro Jesús Teruel

DOI 10.7203/REK.2.2. 8887

233 Gabriel Rivero: Zur Bedeutung des Begriffs Ontologie bei Kant. Berlín, Boston, Walter de Gruyter, 2014, 247 pp. ISBN: 978-3-11-034180-5.

Luciana Martínez.

DOI 10.7203/REK.2.2.8881

\section{Informe}

237 Informe sobre el III Congreso de la Sociedad de Estudios Kantianos en Lengua Española (SEKLE)

Luciana Martínez

DOI 10.7203/REK.2.2.9011

\section{Convocatoria}

242 La recepción de Kant en México

Dulce María Granja Castro; Gustavo Leyva Martínez

Eventos y normas para la publicación

244 Noticias y normas para autores 


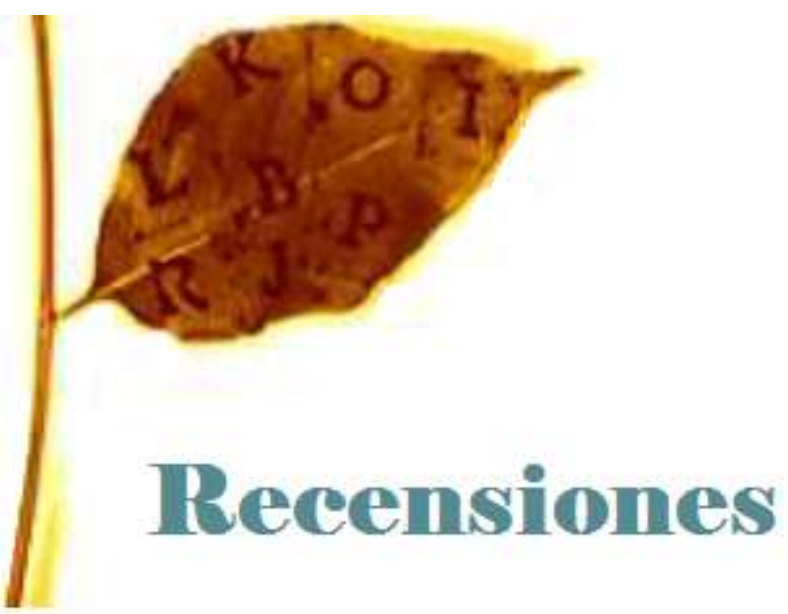




\title{
Claudia Jáuregui, Fernando Moledo, Hernán Pringe y Marcos Thisted (eds.) Crítica y Metafísica. Homenaje a Mario Caimi. Studien und Materialen zur Geschichten der Philosophie. Hildesheim, Olms, 2015, 460 pp. ISBN: 978-3-487-15237-0
}

\author{
ALBA JIMÉNEZ RoDRÍGUEZ
}

El presente volumen, en el que se rinde un cumplido homenaje a la caudalosa trayectoria académica del Prof. Mario Caimi, recoge contribuciones de veinticinco reconocidos expertos del ámbito de la investigación sobre Kant, procedentes de Alemania, España, Francia, Italia, Brasil y Argentina. A lo largo del libro puede entreverse un fecundo diálogo con algunos de los problemas a los que Caimi dirigió su investigación tales como la deducción de las categorías y la doctrina del esquematismo trascendental, el complejo estatuto del tiempo en la filosofía de Kant o el concepto de metafísica en el ámbito de los Fortschritte.

El trabajo de Manfred Baum, titulado "Praktische Erkenntnis a priori in Kants Kritik der praktischen Vernunft", comienza recuperando alguna de las tesis de Hermann Andreas Pistorius sobre la fundamentación kantiana de la moral. Se trata de un autor que, a pesar de haber recibido una atención escasa en el ámbito de la Kant-Foschung, resulta crucial para entender algunos problemas morales así como cuestiones de enorme calado teórico entre los que se encuentran las transformaciones del concepto de metafísica a lo largo del corpus kantiano. En la Crítica de la Razón Práctica, Kant había establecido el hecho paradójico de que aquello que denominamos bueno o malo no debe ser determinado con anterioridad a la ley moral, sino solo de acuerdo a ella y por medio de ella. Por tanto, lo bueno y lo malo, frente a lo que ocurría en la tradición ética desde Platón y Aristóteles, nada tienen que ver con aquello que nos resulta agradable (Wohl) o doloroso (Weh) o con un mal físico $(\ddot{U} b e l)$. Por el contrario, como señala el autor, "nur durch Einstimmung oder Widerstreit mit einem moralischen Gesetz für das Wollen von Handlungen entschieden werden kann" (Jáuregui et al. 2015: 14). Por tanto, el peculiar tipo de objeto que constituye el ámbito de lo práctico (no serían de hecho propiamente objetos, sino acciones de la determinación racional del querer de un sujeto imputable), depende de la legislación universal de la razón sobre nuestra voluntad, entendida como una capacidad de comenzar una serie causal de fenómenos en el mundo.

Lo bueno y lo malo serían en última instancia modi de la categoría de causalidad, como ya se anticipaba en la tercera antinomia de la primera Crítica, con la distinción entre una causalidad eficiente y prolongable infinitamente, que regulaba el mundo sensible, frente a una causa libre concebida como un fundamento de los fenómenos de atribución de responsabilidades morales. El artículo examina asimismo las categorías de la libertad. Según la cantidad un sujeto determina su

${ }^{1}$ Universidad Autónoma de Madrid. 
acción conforme a máximas, preceptos que rigen para una pluralidad o principios a priori de la libertad que vinculan a todo sujeto posible. Según la cualidad contamos con reglas prácticas praeceptive o susceptibles de ponerse en obra, reglas prácticas prohibitivae o de omisión y reglas prácticas exceptivae que incluyen principios de determinación, los cuales suponen una excepción frente a otra regla de conducta. Según la relación, ponemos en común todo principio de determinación de nuestro arbitrio con la personalidad, con el estado de la persona o con la acción recíproca de los estados de diversas personas entre sí. Y, según la modalidad, distinguimos lo lícito de lo ilícito, el deber y lo contrario a deber y los deberes perfectos de los deberes imperfectos. El autor concluye con la idea de que las categorías de la libertad harían ahora prescindible los tradicionales predicados de lo bueno y lo malo pues, conforme a ellas, todas las acciones posibles del libre arbitrio son determinadas como actos de una causalidad racional bajos principios prácticos a priori.

El capítulo "Ontología, epistemología y semántica: sobre la teoría kantiana acerca de la estructura objetual del mundo", de Juan A. Bonaccini, emprende la sugerente tarea de interpretar la Analítica Trascendental como una ontología, en el sentido de una teoría a priori o trascendental de los objetos, en la que el objeto -éste, junto con el uso del concepto de categoría esquematizada y su identificación con los principios del entendimiento puro es quizá el escollo argumentativo de la propuesta más difícil de superar- se define de antemano como una estructura formal del conocimiento. Desde esta interpretación, las categorías mismas se presentan como objetos a priori, y la epistemología, así como la semántica, se presentan como enfoques derivados de la propia ontología. Frente a ciertas lecturas que habían hecho de la filosofía de Kant fundamentalmente una epistemología y hasta una precursora de la filosofía analítica, la propuesta de Bonaccini tiene la perspicacia de devolver la problemática cuestión de la ontología al lugar central que había ocupado en las discusiones de Kant con sus contemporáneos en su intento por esbozar un constructivismo crítico diferenciado del dogmatismo wolffiano. A pesar del conocido distanciamiento de Kant respecto de la ontología tradicional que se arrogaba la tarea doctrinal de erigir un sistema a partir de un conocimiento sintético a priori de cosas en general, Kant imprime un nuevo sentido a la ontología como analítica del entendimiento puro, asociada ahora como puede apreciarse en la reflexión 5936 comentada por el autor, con la filosofía trascendental. Por lo demás, en KU, Kant presenta los predicados ontológicos, entendidos como las únicas condiciones bajo las cuales las cosas pueden convertirse en objetos de conocimiento. Además de proporcionar una visión muy luminosa sobre la distinción entre la Deducción Metafísica y la Deducción Trascendental al hilo de su consideración de la Analítica como ontología, el texto tiene la virtud de dar una salida teórica a la atribución frecuente -en el marco de las investigaciones sobre Kant- de un carácter regulativo al principio del entendimiento puro de relación, esto es, a las Analogías de la experiencia; atribución justificada usualmente en virtud del carácter dinámico del principio. Ciertamente los principios del entendimiento puro completan la operación de aplicación de las categorías a casos particulares del fenómeno (auf besondere Fälle der Erscheinung) ( $\mathrm{KrV}$ A159 B199). Y, en concreto, los Grundsätze dinámicos, como tales, tendrían un carácter meramente regulativo o heurístico. Sin embargo, cada esquema trascendental, con independencia de su vínculo respectivo con categorías matemáticas o dinámicas, cumple ya de hecho esa función referencial de subsunción o aplicación de los conceptos puros a los fenómenos y lo hace en principio con pleno carácter constitutivo. La solución que proporciona Bonaccini al respecto -apelando a la definición que da el propio Kant en la Dialéctica trascendental, cuando afirma que las leyes dinámicas también pueden tenerse por constitutivas en tanto que hacen posibles a priori los conceptos, sin los cuales no tiene lugar experiencia alguna (A664/B692) -es sostener que el carácter regulativo de las analogías puede predicarse sólo en un plano 'metalingüístico'- (Jáuregui et al. 2015: 40).

El trabajo de Christian Bonnet rescata algunos debates apasionantes del neokantismo, partiendo de la tesis de Friedrich Albert Lange que, frente al materialismo reduccionista, había 
presentado una interpretación fisiológica de Kant desde la que confiere un nuevo estatuto a las formas a priori de la sensibilidad o a la problemática distinción entre sensibilidad y entendimiento. El autor hace visible el contexto en el que surge la Geschichte des Materialismus de Lange cuya estrategia teórica inicial consiste en circunscribir la legitimidad del materialismo al ámbito de investigación científica, de modo que dicha tarea científica ponga de manifiesto los límites del propio materialismo. Como Salomon Maimon había hecho, Lange a su vez trae a discusión un momento muy controvertido de la filosofía de Kant visible fundamentalmente a propósito del problema de la cosa en sí, en virtud de la definición que proporciona del concepto de sensación en el principio de las Anticipaciones de la percepción y de su teoría de las facultades. Como Lange observa, causas diferentes pueden provocar una misma sensación y una misma causa puede dar lugar a sensaciones diferentes. No menos problemática resulta la caracterización de la relación de la cosa en sí con los elementos del mundo fenoménico en términos causales, habida cuenta de que la causalidad es ya por definición una estructura eminentemente fenoménica. El autor aborda dos propuestas fundamentales de Lambert: que el mundo de los sentidos es un producto de nuestra organización y que nuestros órganos corporales no son sino imágenes de un objeto desconocido (Jáuregui et al. 2015: 52). El planteamiento de Lange permite revisitar el problema del dualismo aportando nuevas perspectivas para interpretar la división originaria kantiana entre la receptividad y la espontaneidad concebidas ahora como las dos caras de una misma moneda. Por su parte, el recorrido presentado por Bonnet permite asomarse al corazón del trascendentalismo kantiano al introducirnos en el punto álgido de la recusación de las tesis de Kant: el hecho de que los elementos a priori del conocimiento puedan tener un origen empírico y, simultáneamente tener validez objetiva. Esta revisión psicologista de Kant tendrá también profundas consecuencias por lo que atañe al problema de la libertad y de la ley moral a la que de nuevo considera "un élément empirique (...) qui détermine le cours de mes pensées" (Jáuregui et al. 2015: 58).

El texto de Daniel Brauer ofrece una revisión pormenorizada de la recepción hegeliana de la concepción de espacio y tiempo en la estética trascendental kantiana a partir de la cual puede apreciarse cómo la posición de Hegel estaría mucho más próxima a una relectura del papel del tiempo, ligado a la apercepción trascendental o a los procedimientos de síntesis figurativas de la imaginación que a la concepción de espacio y tiempo como formas a priori de la sensibilidad. Respecto a la concepción kantiana circunscrita a las formas puras de la Estética trascendental, Hegel no aceptará ni su condición de intuiciones formales, ni el hecho de que constituyan condiciones de posibilidad para la construcción de juicios matemáticos. En la misma línea, tampoco aceptará su carácter de formas de ordenación sinóptica de la multiplicidad sensible capaces de subsistir con independencia de las propiedades ontológicas de los objetos mismos. Para desarrollar este análisis toma en cuenta las tesis hegelianas vertidas en las Lecciones de Historia de la Filosofía, la Enciclopedia de las Ciencias Filosóficas, la Filosofía Real de Jena o Creer y saber. Frente a las tesis heideggerianas de Ser y Tiempo y de los Prolegómenos para una historia del concepto de tiempo que, en el marco de su crítica a la concepción vulgar, vinculan la propuesta hegeliana a la tradición de la definición aristotélica del tiempo de la Física como el número del movimiento, el autor subraya la pertinencia de evaluar la interpretación hegeliana desde o frente a presupuestos kantianos y newtonianos. Brauer recorre las concepciones del espacio y del tiempo de Hegel en relación con la geometría y la aritmética, así como la definición de los mismos como momentos del movimiento, como 'el concepto mismo', o como 'lo sensible-no sensible', pergeñando algunas líneas maestras muy sugerentes para esbozar una filosofía de la matemática (Jáuregui et al. 2015: 66 ss).

En el trabajo de Wolfgang Carl se ponen en común las reflexiones de Kant en torno al problema de la autoconciencia en las Lecciones de Antropología y en el Apéndice a la Paz Perpetua con las reflexiones actuales en el ámbito, no sólo de la filosofía, sino de la psicología y la neurociencia. La autoconciencia, ligada a la posesión del sentido interno, queda definida como marca 
diferencial del hombre frente a los animales. El artículo tiene la enorme virtud de traducir los problemas del contexto semántico y teórico propio de Kant a un ámbito de reflexión que, como los de T. Burge o Ned Block, parte de presupuestos filosóficos muy diferentes.

La contribución de Bernd Dörflinger, "Diskrete und kontinuierliche Zeit. Ein verborgener Widerstreit bei Kant", resulta de especial interés para trazar las continuidades con uno de los campos de la investigación kantiana en el que la contribución del Profesor Caimi ha resultado muy significativa: el análisis del tiempo en la Crítica de la Razón Pura. Frente al lugar común por el cual la concepción del tiempo en Kant suele limitarse a su definición en la Estética como forma a priori de la sensibilidad, las investigaciones de Caimi han resultado de enorme valía para sacar a la luz la importancia que cobra el tiempo como determinación trascendental en el contexto de la analítica de los conceptos y de los Principios y, en concreto, en la doctrina del esquematismo. El privilegio del tiempo en detrimento del espacio por su condición de forma del sentido interno, ya sugerido en la Estética trascendental, se visibiliza tanto más en la deducción de las categorías con el desarrollo del concepto de la unidad trascendental de la apercepción así como en el Schematismuskapitel en el marco del cual -junto a las definiciones de esquema como procedimiento de la imaginación para proporcionar una imagen a los conceptos puro, intuición sensible, procesos de aplicación de los conceptos a las intuiciones formales, etc.- el esquema se presenta fundamentalmente como una determinación trascendental del tiempo o, como Caimi traduce con mucho acierto, como una determinación trascendental temporal. De la mano de las reflexiones de Gerold Prauss, que a lo largo de su vida ha estudiado profusamente el problema del continuo en el contexto de las determinaciones temporales kantianas, esta contribución supone un punto de partida muy fructífero para elaborar una reconstrucción de la teoría del continuo en Kant (Jáuregui et al. 2015: 96). Precisamente en los principios del entendimiento puro correspondientes a los esquemas de la cantidad y de la cualidad, se pone de manifiesto que el tiempo viene determinado por un orden en el que momentos homogéneos de tiempo se extienden partes extra partes pero, simultáneamente, el tiempo goza de una intensión que proporciona la cualidad o la realitas de los fenómenos y cuya variación puede cifrarse en incrementos gradientes y continuos.

En "El tiempo en Kant: de la Disertación a la Estética trascendental" Jorge E. Dotti lleva a cabo un recorrido muy lúcido sobre las transformaciones del concepto de tiempo en el tránsito a la etapa crítica, tomando como referencia tres hitos fundamentales: la carta a Herz de 1772, las reflexiones del Legado Duisburg y la Estética trascendental de KrV. Es en 1770 cuando espacio y tiempo comienzan a definirse como principios formales de la intuición y cuando el tiempo empieza a conceptualizarse como un fundamento formal del conocimiento que posee cierta preeminencia respecto del espacio. De hecho, la idea de sucesión vinculada al número, aparece ya como condición de inteligibilidad del espacio mismo. Como explica Dotti, este es un momento crucial del desarrollo de la filosofía crítica porque Kant plantea con claridad por primera vez un problema tan antiguo como el platonismo, que hará fortuna en su formulación más perfecta en el capítulo de la deducción trascendental de las categorías de $\mathrm{KrV}$ : ¿cómo hacer ciencia - por tanto eidética- sobre realidades cambiantes y generadas como aquellas de las cuales se ocupa la física? o ¿cómo garantizar que funciones lógicas no extraídas de la experiencia puedan sin embargo aplicarse a ésta cabalmente proporcionando conocimiento objetivo? El texto de Dotti permite trazar un cuadro de aquellos rasgos del concepto de tiempo que permanecen sin modificaciones sustanciales entre los años 1770 y 1781 y, sobre todo, de los rasgos divergentes entre ambos. A lo largo de este desarrollo, Dotti da cuenta del proceso de pérdida de autonomía de la sensibilidad a medida que el tiempo va definiéndose como forma del sentido interno y mostrando su vínculo con la apercepción; desarrollo que guarda una apreciable deuda con las críticas que Schultz, Lambert, Mendelssohn o Sulzer habían realizado a Kant a propósito de la publicación del Habilitationschrift (Jáuregui et al. 2015: 117). 
El texto de Klaus Düsing. "Zeit und Substanz in Kant Kritik der reinen Vernunft", toma como punto de partida la profunda transformación que sufre el concepto de tiempo en Kant frente a otros filósofos de la tradición como Aristóteles o Wolff en el marco de una nueva ontología crítica. El trabajo ofrece una rigurosa interpretación del significado de los conceptos de sustancia y de tiempo en la teoría del conocimiento kantiana. El concepto de sustancia, como toda función lógica de la tabla categorial, recibe su estructura formal de las funciones judicativas las cuales reciben a su vez su fundamento de la unidad sintética de la apercepción. El autor explica el papel del esquema de la permanencia como determinación trascendental temporal de relación en su carácter de tertium quid homogéneo tanto con la categoría como con las formas a priori de la intuición para concluir con una original evaluación del problema de la permanencia en el conocimiento específico de los objetos de la naturaleza y en la autodeterminación del yo empírico (Jáuregui et al. 2015: 162).

El artículo de Dietmar H. Heidemann, "Zwei Formen der Identität in Lockes Theorie der Person", estudia la respuesta de Locke al problema de la identidad personal a través del recurso teórico de la autoconciencia. El trabajo de Heidemann pone de manifiesto cómo la teoría lockeana se construye en forma de diálogo con la tradición que arranca en Platón, según la cual la respuesta al problema de la identidad se debate entre dos opciones fundamentales: dar cuenta de la identidad bien a partir de la identidad del alma bien de la identidad del cuerpo. El texto se articula en torno a una división establecida por Locke entre diferentes concepciones de la identidad marcadas por respectivas determinaciones temporales. El respecto retrospectivo de la identidad da lugar a una identidad del recuerdo que permite atribuir conductas del pasado relevantes desde el punto de vista jurídico y moral. El respecto prospectivo da lugar a una identidad a futuro a partir de la cual pueden imputarse conductas morales y jurídicas que nos hacen merecedores de castigos y recompensas en virtud de nuestras acciones pasadas (Jáuregui et al. 2015: 165). El trabajo alumbra uno de los presupuestos fundamentales de la solución de Locke al problema de la identidad personal. Y es que, frente a otros autores, el problema de la identidad no es un problema estrictamente gnoseológico que involucre la cuestión de la permanencia de la sustancia como entidad ontológica, sino un problema práctico tan relevante como el problema de la imputación.

El texto de N. Hinske, titulado "Kants Beantwortung der Frage: Was ist Aufklärung?", ofrece una perspectiva muy novedosa sobre la distinción formulada por Kant entre el uso público y el uso privado de la razón, a partir de una de las cruces philosophorum de la filosofía kantiana: la isomorfía lógico-estructural entre la política, el derecho y la religión. El artículo visibiliza el contexto en el que se formula la famosa pregunta de la Berlinische Monatsschrift aludiendo a la contribución de Ernst Ferdinand Klein Über Denk- und Druckfreiheit an Fürsten, Minister und Schriftsteller que anticipa la distinción kantiana entre el uso público y el uso privado de la razón y muestra las líneas de continuidad con las anotaciones de Naturrecht Feyerabend correspondiente a las lecciones del semestre de verano precisamente del año 1784 .

El trabajo de Claudia Jáuregui, titulado "Kant y Hume: sobre los alcances de la demostración del principio de la segunda analogía de la experiencia", despliega una cuidada y rigurosa argumentación que pone de manifiesto cómo, por un lado, los problemas encerrados en la crítica a la noción de causalidad de Hume no pueden ser resueltos con las herramientas teóricas empleadas por Kant en la demostración de la segunda analogía de la experiencia y, simultáneamente, las observaciones de Kant al hilo de dicho principio puro del entendimiento desbordan el marco teórico establecido por Hume a propósito del problema de la causalidad. Jáuregui distingue cuatro principios de la propuesta kantiana, de los cuales, los dos primeros (la ley de la sensibilidad según la cual el tiempo anterior determina necesariamente al siguiente y todo lo que ocurre presupone algo a lo cual sigue según una regla) son desatendidos por el análisis humeano, mientras que los dos últimos (que la forma de las leyes causales particulares impone que cierto fenómenos son la causa de otro tipo 
de fenómenos y que a causas similares le siguen efectos similares) son el verdadero objeto de análisis de Hume consagrado a la justificación de estas leyes (Jáuregi et al. 2015: 184 ss). La necesidad de demostrar leyes empíricas particulares desborda el ámbito de la segunda analogía e implica la remisión a principios regulativos de los cuales Kant no da cuenta sino en la Crítica del Juicio.

El trabajo de Heiner F. Klemme, "Kants Rezension von Johann Heinrich Schulz. Versuch einer Anleitung zur Sittenlehre für alle Menschen ohne Unterscheid der Religionen", analiza un aspecto muy novedoso de la recensión kantiana sobre el texto de Schulz titulado Versuch einer Anleitung zur Sittenlehre für alle Menschen, ohne Unterscheid der Religionen, a saber: la deducción del concepto de libertad en conexión con los planteamientos de la Fundamentación de la Metafísica de las Costumbres. Frente a la idea avalada también por las lecturas clásicas de la tercera antinomia de $\mathrm{KrV}$ las cuales consideran que el fundamento de la idea de una voluntad libre reside en el carácter inteligible y el uso teórico de la razón, Klemme subraya la importancia de un "unmittelbar praktischen Gebrauch" de la idea de libertad (Jáuregi et al. 2015: 199).

La contribución de Claudio La Rocca sobre "L'architettonica nella Methodenlehre della Critica della ragion pura" da continuidad a cierta línea de trabajo emprendida con el objetivo de recuperar la importancia de la Doctrina trascendental del método, frente a la desatención sufrida en el marco de las investigaciones kantianas o de su reducción a una consideración de la misma como forma de la argumentación trascendental. El texto recupera y arroja nueva luz sobre la división de la filosofía trascendental en crítica, disciplina, canon y arquitectónica reiterada en una reflexión de los años 1775 y 1778 y en una carta a Marcus Herz de 1776.

El artículo de Daniel Leserre, "La función semántica del lenguaje en la deducción trascendental de la Crítica de la Razón Pura", establece un fecundo diálogo con una de las tesis fundamentales establecidas por Mario Caimi en su obra Leçons sur Kant. La déduction trascendantale dans la deuxième édition de la Critique de la raison Pure: la idea de que la deducción trascendental provee un fundamento a la función semántica entendida como capacidad de significar o referir objetos, no sólo de los conceptos puros, sino también del lenguaje en general. El texto de Caimi tenía como objetivo prioritario subrayar el papel desempeñado por la apercepción, demostrar que las categorías no son conceptos vacíos, es decir, que se aplican a objetos empíricos y explicar el método sintético de exposición de la argumentación kantiana. El texto articula la tesis a partir de los siguientes pasos argumentales. En primer lugar, establece la idea de que la Bedeutung concierne a los objetos y debe proceder a través de una deducción. En segundo lugar, que algunos conceptos puros pueden tener un uso a priori y que dicho uso requiere siempre de una deducción. En tercer lugar, que la función de la deducción trascendental consiste precisamente en explicar cómo dichos conceptos pueden referirse a priori a objetos no extraídos de la experiencia, por tanto, en determinar el significado a priori de los conceptos puros. De aquí puede concluirse que la deducción proporciona una fundamentación a la función semántica de las categorías y por extensión al lenguaje en general, abriendo una línea de interpretación de la primera críctica muy fecunda en el campo de la filosofía del lenguaje (Jáuregi et al. 2015: 240 ss).

El artículo de Bernd Ludwig, "Notwendigkeit ist nichts als jene Existenz, die durch die Möglichkeit selbst gegeben ist", ofrece una interesante perspectiva sobre la influencia de la tesis leibniziana acerca del argumento ontológico y la corrección que hace a la prueba cartesiana sobre la existencia de Dios en tres textos: Animadversiones in partem generalem Principiorum Cartesianorum, Meditationes de Cognitione, Veritate et Ideis y De Synthesi et Analysi universali seu ars inveniendi et judicandi. Es conocida la crítica kantiana al argumento ontológico por la cual afirma que el ser no es un predicado real. Frente al uso lógico del 'es' como estructura copulativa que permite conectar un sujeto y un predicado, la existencia designa la posición absoluta de una cosa y 
por tanto no es una propiedad de los objetos que caen bajo un concepto, sino una propiedad de los objetos mismos. Le necesidad de los objetos trascendentales no radica en su existencia como objetos, sino en su necesaria representabilidad por la razón. La causa libre no puede ser conocida, pues se trata precisamente de una entidad no sujeta a la conexión fenoménica temporal sobre la cual puedan operar sinópticamente las formas a priori de la sensibilidad y ser subsumidas bajo conceptos, pero sí es necesaria en sentido práctico, si queremos atribuir responsabilidad jurídico-moral a los sujetos por sus acciones. Frente a Leibniz y en general a la tradición escolar alemana, Kant establece una diferencia fundamental entre la realidad objetiva de las ideas trascendentales y la necesidad de los objetos.

La contribución de Macarena Marey, "El rol sistemático de los fines en la metafísica de las costumbres", alumbra las implicaciones del hecho de que las líneas fundamentales de la filosofía jurídico-política de Kant, como prueban algunas de sus reflexiones ya desde 1764, se establecieron con anterioridad a la fijación del lugar sistemático que debía ocupar el derecho en el marco más amplio de su filosofía práctica. A juicio de la autora, el papel del concepto de fin resulta fundamental para cifrar esta localización sistemática por cuanto desarrolla la tarea de guiar la diferenciación del método respectivo de las doctrinas de la virtud y del derecho en que se divide la Metafísica de las Costumbres. El rechazo del teleologismo en su versión perfeccionista y eudemonista resulta además fundamental para la gestación de la revisión kantiana del iusnaturalismo moderno.

En el artículo "Nöthigung, necessitatio, necesitación. Sobre el significado de un concepto kantiano de la filosofía práctica", Fernando Moledo lleva a cabo un estudio sobre las derivas filológicas del término necessitatio que permite reevaluar el significado filosófico del problema de la obligación en la filosofía práctica kantiana. El autor toma como referencia de partida la definición que proporciona del término Nöthigung el Diccionario para el uso sencillo de los escritos kantianos de Schmid, el cual identifica la obligación con los sentidos restringidos de obligación moral y obligación práctica, con el Diccionario Enciclopédico de términos kantianos de Mellin que lo define en conexión con el imperativo categórico, la traducción al latín de J. Ch. Zwanziger por el término necessitatio, y la puesta en común de F.G, Born de la Nöthigung con la coactio. Moledo retrotrae el uso de este concepto a la Metafísica de Baumgarten donde éste introduce el término para referirse al cambio de una sustancia de lo contingente a lo necesario, recogiendo a su vez el uso que Leibniz hiciera del vocablo nécessitation para distinguir entre el carácter libre o prestablecido de las sustancias. De igual modo que en la antítesis de la tercera antinomia, Kant reconoce que la serie de efectos subalternos debe recibir su fundamento de un tipo especial de causalidad que corte esta concatenación infinita de causas, por su parte, Leibniz había afirmado anteriormente que el principio de razón suficiente tiene que tener su término en un intelecto divino. Pero ya Leibniz se planteaba entonces cómo hacer compatible dicha determinación inexorable de los planes divinos con la existencia de acciones libres por parte del entendimiento finito. La nécessitation es para Leibniz justamente el tipo de necesidad que se da en las sustancias cuando son libres. En continuidad con esta definición, la Nöthigung indica para Kant el tipo de necesidad que se establece para los seres finitos contingentes en los que la coincidencia de su voluntad con los fundamentos objetivos de determinación del obrar puede darse o no darse, en la medida en que el ser humano se ve condicionado por Triebfedern o motores impulsores de carácter sensible que pueden dar lugar a máximas que no coincidan con los principios de la moralidad. El deber impone una necesidad ante hechos que son contingentes, en el sentido de que es posible libremente elegir o no elegir observar los principios que la sustentan. En el caso de la voluntad santa, por el contrario, el único motivo impulsor es el respeto mismo hacia la ley. El autor establece una triple caracterización de la Nöthigung en correspondencia con los tres tipos de imperativos (morales, técnicos o pragmáticos) lo que le sirve para refutar la identificación de Schmid entre la moralische Nöthigung y la praktische Nöthigung, poniendo de manifiesto que la obligación moral es sólo un tipo de la obligación práctica. Para terminar, frente a la identificación de Born entre 
necessitatio y coacción, Moledo muestra que la coacción para Kant no es sino un tipo específico de necessitatio (Jáuregi et al. 2015: 305).

La contribución de Hernán Pringe, "Metafísica, lógica y probabilidad cuánticas", proporciona una respuesta audaz a la pregunta por la posibilidad de establecer principios metafísicos en sentido kantiano para la metafísica cuántica. El argumento de Pringe toma como punto de partida la aplicación de la distinción kantiana entre lo constitutivo y lo regulativo a la distinción correlativa entre la parte general y la parte especial de la metafísica de la naturaleza. El autor comienza demostrando que no es posible dar con principios constitutivos de la naturaleza de modo que obtengamos a partir de ellos principios metafísicos de la física cuántica. Los objetos cuánticos no poseen validez constitutiva, pero sí regulativa, en tanto que deben concebirse -no como objetos de la experiencia posible- sino objetos metacontextuales. Esto se explica porque la verificación de los fenómenos cuánticos no puede tener lugar en todo contexto, pero dado un fenómeno cuántico determinado, podemos establecer la probabilidad de cualquier otro fenómeno a través de una regla de unificación (Jáuregi et al. 2015: 312).

El texto de Alberto Rosales, "La teoría de la apercepción en el capítulo de los paralogismos", retoma la cuestión de la circularidad de la autoconciencia. El debate atiende a dos posiciones fundamentales: una que afirma la imposibilidad de la autoconciencia por su caída en un regreso al infinito y la otra, representada paradigmáticamente por Düsing, según la cual dicha tesis implica una concepción errónea de la autoconciencia. El artículo aborda las críticas de Kant a la psicología racional y concluye que el presunto círculo de la autoconciencia no se refiere al hecho de que ésta se presuponga a sí misma, sino más bien al hecho de tener conciencia de la actividad unificadora de la propia conciencia.

El trabajo de Jacinto Rivera de Rosales, "Kant: gusto y reflexión. La presencia del concepto en la experiencia estética", da cuenta exquisitamente del papel que juega el concepto en la estética kantiana. El autor explica la especificidad de los juicios estéticos frente a los juicios lógicos. Frente a los juicios lógicos en los que subsumimos los objetos empíricos bajo conceptos en virtud de una finalidad concreta para poner lo real a disposición del pensar y de nuestros intereses morales y prácticos, el juicio estético no descansa en conceptos, sino en el sentimiento de placer que propicia el libre juego de la imaginación, ya no entendida, tal como sucede en la Analítica de los principios, como una facultad mecánica que hace posible los procedimientos de subsunción de las intuiciones formales bajo las funciones lógicas, sino como un movimiento que procura la mediación entre lo sensible y lo inteligible en virtud de un particular juego entre lo prerreflexivo y lo reflexivo. Lejos de lo que sugieren ciertas interpretaciones de Kant, lo estético requiere de la trama categorial con la que habitualmente nos pertrechamos de los supuestos teóricos que nos permiten comprehender el mundo, pero, precisamente para suspenderlo y posibilitar un singular cambio de mirada despojado de todo interés pragmático (Jáuregi et al. 2015: 346).

El artículo de M. Ruffing, "Gedanke, Sprache, Ton - Bemerkungen zu Kants Sprachkritik", expone algunos elementos sobre las relaciones entre el lenguaje y el pensamiento. Para Kant todas las lenguas son signos del pensamiento. La contribución de Ruffing parte de las consideraciones sobre la concepción instrumentalista del lenguaje de la Antropología en sentido pragmático. La autocomprensión humana y la comunicación exitosa llevan implícita una idea de comunidad que a su vez está presupuesta en la idea de una razón práctica. Kant lleva a cabo una crítica lingüística orientada fundamentalmente a los filósofos profesionales. Entre los intérpretes de Kant, prevalece la idea de que Kant en su filosofía teórica no hizo justicia a la importancia del lenguaje y que por tanto no tiene una teoría propia al respecto. A partir del siglo XX, sin embargo, hubo una recuperación de la filosofía del lenguaje en relación con la semiótica trascendental. En esta rehabilitación de la 
filosofía del lenguaje kantiana lo importante son las reflexiones sobre el mismo en el ámbito de la filosofía moral. Se postula entonces una suerte de concepción normativa del lenguaje que permite distinguir un empleo adecuado o inadecuado de este. Este uso debe ser adecuado a la capacidad moral del hombre. El mencionado uso normativo del lenguaje está presidido por el mandato de veracidad y su transgresión implica la pérdida de nuestra condición de hombres. Lo que propone en su doctrina de la virtud es una relación libre del sujeto hablante con las cosas en el mundo. El lenguaje conlleva una cierta autocomprensión moral. El hombre se convierte en una Sprachmaschine cuando no obedece este mandato (Jáuregi et al. 2015: 364). El filósofo contrae una obligación especial en lo que se refiere al trato con el lenguaje y, sin embargo, a menudo falla en la transmisión veraz de los pensamientos; tiene pues un riesgo profesional de carácter moral debido a su tono. El tono implica precisamente presentarse al mundo con autoconfianza; provoca una disposición que afecta al ánimo del que lee. El uso práctico moral de la razón puede determinar el tono. A través de un cierto tono distinguido los filósofos encubren la falta de comunicabilidad de sus reflexiones filosóficas o bien directamente diseminan errores traicionando su compromiso con la verdad.

En “Transformaçoes na filosofía da história kantiana na década de 1780" Ricardo Terra pone la mirada en la fascinante confluencia filosófica producida en el año 1784, momento en el que concluye el manuscrito de la Fundamentación de la Metafísica de las Costumbres, las lecciones de derecho natural (Naturrecht Feyerabend) y Moral Mrongovius II, las Ideas para una historia universal en clave cosmopolita y ¿Qué es la Ilustración? Dicho año supone un momento clave en el desarrollo de la concepción teológico-crítica de la historia y de la distinción entre derecho y ética. En el texto se dan cita asimismo algunas consideraciones sobre la cuestión de las razas a propósito de Las diferentes razas humanas publicado en 1775 como anuncio del curso de geografía física, Determinación del concepto de una raza humana de 1785 y Sobre el uso de principios teológicos en teología, donde Kant responde a las críticas de Forster tratando de resolver problemas como la distinción entre los conceptos de raza, variedad y especie. El autor considera con Bordoni que la Fundamentación de la Metafísica de las Costumbres, así como Naturrecht Feyeranbend y Moral Mrongovius II publicados en el volumen XXVII de la Academia constituyen "drei Variationen eines desselben Gedankes" y subraya también la necesidad de poner en común sus reflexiones con Idee donde acaban de formularse conceptos centrales para la filosofía jurídica de Kant (Jáuregi et al. 2015: 382). Los textos de estos años realizan contribuciones tan importantes como anticipar la distinción entre ética y derecho tal y como será perfilada posteriormente en 1797.

El trabajo de Marcos A. Thisted sobre "La Weisheitslehre en los Fortschritte der Metaphysik" recobra una tesis fundamental de Caimi según la cual la Weisheitslehre ocupa el lugar que había dejado vacante la metafísica dogmática leibnizo-wolffiana. El artículo estudia las implicaciones de la aparición del término en la Crítica de la Razón Práctica y su desarrollo sistemático en Los Progresos de la Metafísica. La nueva definición de la metafísica proyectada en esta obra opera como principio de organización sistemática constituido en tres estadios de la razón pura (el de lo sensible, el del tránsito de lo sensible a lo suprasensible y el de lo suprasensible) correlativos a la doctrina de la ciencia, la doctrina de la duda y la doctrina de la sabiduría, la cual tiene su principio de articulación en el bien supremo y comprendería una teología moral que sustituiría a la teología racional, una teleología moral que sustituiría a la cosmología racional y una psicología moral que reemplazaría a una psicología racional.

La excelente contribución de María Jesús López Lobeiras parte del análisis de las definiciones de juicio en la deducción de la segunda edición de la Crítica de la Razón Pura, así como en algunos pasajes de los Prolegómenos a toda metafísica futura. El artículo tiene como punto clave el desarrollo de la tesis de que entre la exposición de la deducción de las categorías en la primera y la segunda edición se produce un salto de la noción de objeto a la noción de objetividad, vehiculado por 
la relación que establece Kant entre en el juicio y la apercepción en la segunda versión de la Deducción.

Husserl se había referido a Kant como al 'protofundador' de una filosofía de nuevo cuño, a la filosofía moderna como a aquella cuya su secreta aspiración era la fenomenología y al sistema kantiano como el primer ensayo de alcanzar una filosofía universal con el carácter de ciencia estricta. El libro se cierra pues, de la mano de Roberto J. Walton, abordando una interesantísima cuestión como es "La articulación kantiana de la fenomenología de Husserl" que, en la línea de las demás contribuciones, corresponde al rigor con que el homenajeado ha abordado los problemas de la filosofía kantiana en su dilatada carrera. 